\title{
Performance of MIMO FMCW radar in detecting small vessels
}

\begin{abstract}
This paper evaluated the performance of range detection of a MIMO FMCW radar system detecting small vessels in maritime environments, through numerical simulations. The targets were modeled as Swerling1 targets, which resemble target with slow-changing radar cross sections (RCS). A MIMO FMCW system utilizing two transmitting antennas emitting two FMCW signals in different frequency bands, and two receiving antennas for MIMO processing - is proposed. In the MIMO scheme, a waveform design comprises of two subbands with an interval band, is suggested to avoid co-band interference between the two signals emitted from two transmitting antennas. At the receiver, the receiving signals were combined by means of spectrum averaging, before implementation of peak detection to estimate the target range. The performance of the proposed system is observed in terms of probability of range error, simulated over a range of signal-to-noise-ratio (SNR). Simulation results indicated that MIMO processing yields approximately $3 \mathrm{~dB}$ improvements of probability of range error against SNR, compared to SISO case observed at $20 \%$ range error probability. In addition, the effects of interval band deployment on the quality of beat frequency was also discussed.
\end{abstract}

Keyword: FMCW; Radar; Swerling; SISO; MIMO; Range 